\title{
Automation of Differential Blood count
}

\author{
Neelam Sinha and A. G. Ramakrishnan \\ Bio-Medical Lab \\ Department of Electrical Engineering \\ Indian Institute of Science \\ Bangalore, 560012 INDIA \\ Tel : $+91-802932935$ \\ \{neelam,ramkiag\}@ee.iisc.ernet.in
}

\begin{abstract}
A techmique for automating the differential count of blood is presented. The proposed system takes as input, color images of stained peripheral blood smears and identifies the class of each of the White Blood Cells (WBC), in order to determine the count of cells in each class. The process involves segmentation, feature extraction and classification. WBC segmentation is a two-step process carried out on the HSV-equivalent of the image, using K-Means clustering followed by EM-algorithm. Features extracted from the segmented cytoplasm and nucleus, are motivated by the visual cues of shape, color and texture. Various classifiers have been explored on different combinations of feature sets. The results presented here are based on trials conducted with normal cells. For training the classifiers, a library set of 50 patterns, with about 10 samples from each class, is used. The test data, disjoint form the training set, consists of 34 patterns, fairly represented by every class. The best classification accuracy of $97 \%$ is obtained using Neural networks, followed by $94 \%$ using SVM.
\end{abstract}

Keywords: Differential blood count, Cell segmentation, EM algorithm

\section{INTRODUCTION}

Human peripheral blood consists of 5 types of White Blood Cells (WBC) called lymphocytes, monocytes, eosinophils, basophils and neutrophils [2]. Differential blood count (DBC) is carried out to calculate the relative percentage of each type of WBC, since it helps in diagnosing many ailments. High neutrophil count could suggest cancer, while high lymphocyte count suggests AIDS. High monocyte and eosinophil count usually point at bacterial infection. Thus DBC forms an important statistic of one's health status. A typical blood smear consists of WBC's, red blood cells, platelets, plasma and cell fragments. The automation of DBC involves segmentation, feature extraction and classification, followed by a counter to keep track of the number of cells counted in each class. Some of the techniques proposed to accomplish this task, are explained in the following section.

\section{SURVEY OF EXISTING METHODS}

Bikhet et al. [7] have reported segmentation and classification of the 5 types of WBC's in peripheral blood using gray images of blood smears. Hierarchical thresholding is used to localize the WBC's. The features extracted are the areas of nucleus and cytoplasm, average gray level, circularity measure and the ratio of nucleus to cell area. Classification accuracy of $90 \%$ is reported on 71 cells. The nature of the cells as being healthy or diseased isn't mentioned. Besides, the classifier used has not been disclosed. Ongun et al. [11] have worked on color smear images, containing both peripheral and immature cells. WBC's are segmented by morphological preprocessing combined with fuzzy patch labeling. Shape features are areas of cell and nucleus, ratio of nucleus to overall cell area, cell perimeter, compactness and boundary energy of the nucleus. Texture features include contrast, homogeneity and entropy derived from the gray-level co-occurrence matrix. Color histogram, mean and standard deviation of the color components in CIE-Lab domain, form the color features. This 57-dimensional feature set is used for classification using various classifiers, with a peak performance of $91 \%$ using SVM. The condition of the cells as being healthy or diseased is not mentioned. Park [5] has reported segmentation and classification carried out on low resolution gray images of immature cells. The feature set consists of shape, texture and statistical color features. Neural nets have resulted in an accuracy of $70 \%$. The performance of the DBC-system depends most on the quality of segmentation, since the subsequent steps in the analysis depend on it. Color information could be utilized for more reliable segmentation. Besides, to make the system automatic, it is necessary to make the segmentation free of parameter-tuning. The system is followed by extraction of simple features, motivated by visual cues, for classification.

\section{SySTEM OVERVIEW}

The proposed system aims at distinguishing between the five classes of mature WBC's, namely lymphocyte, monocyte, eosinophil, basophil and neutrophil, in order to automate the process of differential blood count. The input to the system is a digital image of blood smears of healthy subjects and the output is the differential count of the cells.

The main stages of the proposed system are: (i) Acquisition 
TENCON $2003 / 548$

(ii) Segmentation (iii) Feature Extraction and (iv) Classification. The process of acquisition of images is given below. The remaining blocks of the system are explained in subsequent sections.

Acquisition: The color images of the blood smear on the slide were captured using a digital camera mounted on the microscope. The database consists of images of peripheral blood smear slides of healthy subjects, stained using MayGrunwald-Giesma (MGG) stain. The database was obtained from the collaborating clinic of the University of Kaiserslautern, Germany. Typical size of the images handled is $1000 \times 1300$. Each image contains one or more cells of the same or different types. The collection consists of about 75 lymphocytes, 23 monocytes, 21 eosinophils, 12 basophils and 55 neutrophils. In order to give equal weightage to each of the classes, comparable number of samples from each class have been chosen for training as well as for testing.

\section{SEgMENTATION SCHEME}

The performance of any segmentation technique is limited by one or more of the following factors: significant case-specific distinctions in blood smear preparation, smear staining and image acquisition conditions. Most techniques proposed earlier are sensitive to the right selection of parameters such as, threshold, mask-size and initial contour [8], [10], [9]. Also, the assumption of circular shape is untenable most of the times. Hence, we devise a robust technique free from the above assumptions as well as the need for user-interaction to tune parameters. In this paper, we propose a two-part segmentation scheme that enables us to distinguish the WBCcytoplasm and nucleus from the input image of a blood smear.

The objective of segmentation is to extract the WBC's from the background cells as well as to distinguish between the cytoplasm and the nucleus. We first locate the nuclei of the cells using K-Means clustering following which a rectangular region that encompasses the entire cell is cropped. Subsequent processing is carried out on these sub-images each of which is assumed to contain only one WBC. K-Means clustering followed by EM-algorithm are used to get the final segmentation. Protrusion of neighboring cells is removed using connected component analysis. The image is converted to its HSV equivalent using,

$$
\begin{aligned}
& H=\cos ^{-1}\left[\frac{\frac{1}{2}[(R-G)+(R-B)]}{\left[(R-G)^{2}+(R-B)(G-B)\right]^{\frac{1}{2}}}\right] \\
& S=1-\frac{3}{R+G+B} \min (R, G, B) \\
& V=\frac{1}{3}(R+G+B)
\end{aligned}
$$

Each pixel in the image is represented by a vector of $3 \mathrm{com}$ ponents namely, $\mathrm{H}, \mathrm{S}$ and $\mathrm{V}$. The S-component, as it plays a more conspicuous part, is given more weightage as compared to the other two. K-Means clustering is performed on this collection of vectors. In our trials, we have used 5 clusters.

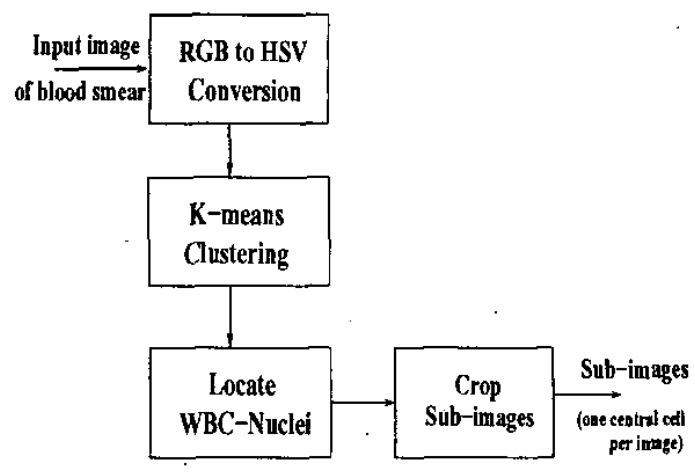

(a)

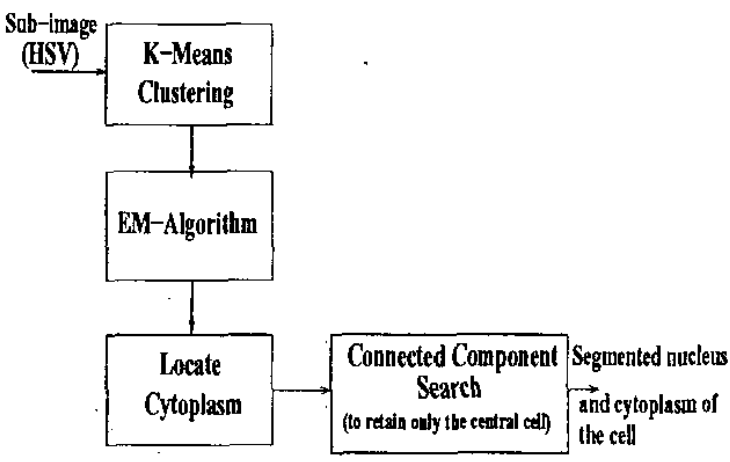

(b)

Figure 1. Overview of the segmentation scheme (a) Generation of sub-images containing single cells (b) Segmentation of nucleus and cytoplasm

Centroids are initialized by finding the mean vector and looking for those $\mathrm{K}$-vectors that are farthest from the mean. Euclidean distance in the feature space is used as the measure of dissimilarity. The convergence criteria is that the difference in the centroids in successive iterations is less than a pre-defined threshold. At the end of this run, we get a class label for each of the pixels, and the centroids for each of the classes. A priori knowledge tells us that the centroid with maximum value of saturation, corresponds to the nucleus. We then crop a rectangular region, surrounding the nucleus, of sufficient area so as to enclose the entire cell. Thus sub-images, each containing only one WBC are obtained. Further processing involves two steps: (i) Initial estimation of parameters using K-Means (ii) Refinement of parameters using EM.

\section{Initial Estimation using $K$-Means}

Each sub-image is separately processed. K-Means clustering is repeated on the smaller dataset to obtain tighter clusters. At the end of this, we obtain a class-label for each pixel and the centroids for each class. Each cluster is modeled by a Gaussian distribution. The parameters are initialized using 


\section{Medical Image Processing / 549}

the clustering obtained by the K-Means algorithm. For the $k$ th cluster, the mean is given by,

$$
\mu_{k}=\frac{1}{n_{k}} \sum_{i=1}^{n_{k}} \mathbf{x}_{\mathbf{i}}
$$

where, $\mathbf{x}_{\mathbf{i}}$ is every 3-D vector that belongs to the $k$ th cluster, $\mu_{k}$ is the mean vector and $n_{k}$ is the number of vectors in the $k$ th cluster.

Since the three features $\mathrm{H}, \mathrm{S}$ and $\mathrm{V}$ are independent, the offdiagonal elements of the covariance matrix are taken as zero. Hence only the variance of each of the dimensions need to be computed. For the $k$ th cluster, the $d$ th diagonal element of the covariance matrix is given by:

$$
C_{d d}^{k}=\frac{1}{n_{k}} \sum_{i=1}^{n_{k}}\left(x_{i d}-\mu_{k d}\right)^{2}
$$

where, $n_{k}$ is the number of vectors in the $k$ th cluster, $x_{i d}$ is the $d$-th dimension of the $i$ th vector and $\mu_{k d}$ is the $d$ th dimension of the mean vector of cluster $k$. These parameter values are refined in the subsequent step. The EM-algorithm is employed as follows.

\section{Parameter refinement using EM}

The EM algorithm [6] consists of two steps: an Expectation step, followed by a Maximization step. The Expectation is with respect to the unknown underlying variables, using the current estimate of the parameters and conditioned upon the observations. The Maximization step then provides a new estimate of the parameters. These two steps are iterated until convergence.

The E Step :-The E step computes the probability $S_{i k}$ associated with the labeling the $i$ th pixel, $x_{i}$ as belonging to the $k$ th cluster,

$$
S_{i k}=\frac{1}{2 \pi\left|\mathbf{C}^{k}\right|^{\frac{3}{2}}} e^{\left.-\frac{1}{2}-\mu_{h}\right)^{T}\left(\mathbf{C}^{h}\right)^{-1}\left(\mathbf{x}_{i}-\mu_{h}\right)}
$$

where $C^{k}$ is the covariance matrix associated with cluster $k, \mu_{\mathrm{k}}$ is the mean vector of cluster $k, i$ and $k$ take values $1,2 \ldots N$ and $1,2 \ldots K$, respectively. Here $N=$ width $\times$ height and $K=$ Number of clusters.

The M step:- -The M-step refines the model parameters given the clustering arrived at E-step. The weighted mean of the $k$ th cluster is updated as:

$$
\hat{\mu}_{k}=\frac{\sum_{1}^{n_{k}} S_{i k} x_{i}}{\sum_{1}^{n_{k}} S_{i k}}
$$

The weighted variance of the $d$ th feature in the $k$ th cluster is updated as: .

$$
\hat{C}_{d d}^{k}=\frac{\sum_{i=1}^{n_{k}} S_{i k}\left(x_{i d}-\hat{\mu}_{k d}\right)^{2}}{\sum_{i=1}^{n_{k}} S_{i k}}
$$

where $x_{i d}$ is the $d$ th dimension of the $i$ th vector and $\hat{\mu}_{k d}$ is the $d$ th dimension of the mean vector of cluster $k$.
Both $\mathrm{E}$ and $\mathrm{M}$-steps are carried out iteratively. The convergence criteria is taken as;

$$
\left|\hat{\mu}_{k}^{(n+1)}-\hat{\mu}_{k}^{(n)}\right|<T \text { Theshold }
$$

Thresholding each of the distributions results in one region being captured in one distribution. A priori knowledge helps us associate nucleus with Gaussian distribution with the highest level of saturation, while cytoplasm is identified as the distribution with maximum number of pixels in immediate contact with the nucleus.

\section{FEATURE EXTRACTION}

Features for discriminating between different cell classes are devised based on domain knowledge of human experts. The features considered are based on (i) Shape (ii) Color (iii) Texture.

\section{Shape features:}

Shape descriptors [3] are a set of numbers that describe a given shape. We use the binary masks of the nucleus and cytoplasm to compute these features. The features considered are eccentricity of the nucleus and cytoplasm contours, compactness of the nucleus, area-ratio and the number of nucleus lobes. Eccentricity is defined as the ratio between the major and minor axes, while compactness is defined as the ratio of area to square of the perimeter. Area ratio is taken as the ratio of the number of pixels that make up the cytoplasm to the ones that make up the nucleus. The number and structure of nucleus lobes is one of the prominent features used to identify the class of the WBC. A declustering technique involving the computation of the negative distance transform followed by watershed algorithm is used [1]. The advantage of using this method is that the contour information is not lost as would have been if one used morphological operations of open-close, for declustering.

\section{Color features:}

The color features are extracted from the segmented nucleus and cytoplasm. The average value of each of the color components $(R, G$ and $B)$ of the nucleus and those of the cytoplasm are computed.

$M e a n_{C}=\frac{1}{N} \sum_{1}^{N} C_{i}$ where $\mathrm{N}$ is the total number of pixels in the region of interest (either nucleus or cytoplasm) and $C_{i}$ is the corresponding color (either $\mathrm{R}$ or $\mathrm{G}$ or $\mathrm{B}$ ) component of the $i$ th pixel.

\section{Texture features :}

Texture features [12], [4] are computed only for the cytoplasm. The texture of the cytoplasm is visually distinct across the various classes, but it is not so for nucleus. The texture features used are based on computations of Gray-Level cooccurrence matrix (GLCM) and Autocorrelation matrix. The features based on GLCM are energy, entropy and correlation. The features based on autocorrelation matrix are coarseness and busyness. Gray-scale co-occurance matrix $\mathbf{P}_{\mathbf{d}}$ is ob- 
tained by

$\mathbf{P}_{\mathrm{d}}=\mid((r, s),(t, v): I(r, s)=i, I(t, v)=j \mid$

The features computed are :

- Energy $=\sum_{i} \mathbb{\Psi}_{j} \quad \mathrm{~d}^{2}(i, j)$

- Entropy $=-\sum_{i} \sum_{j} \mathbf{P}_{\mathbf{d}}(i, j) \log \mathbf{P}_{\mathbf{d}}(i, j)$

- Correlation $=\sum_{i} \sum_{j} \frac{(i-\mu)(j-\mu) \mathbf{P}_{\mathrm{d}}(i, j)}{\sigma_{z z} \sigma_{y}}$

where $\mu$ is the mean of $\mathbf{P}_{\mathbf{d}}$ and $\sigma_{\boldsymbol{x}}$ and $\sigma_{y}$ are the standard deviations of $\mathbf{P}_{\mathbf{d}}(\mathbf{x})$ and $\mathbf{P}_{\mathbf{d}}(\mathbf{y})$ respectively.

Autocorrelation matrix $\rho(x, y$ is computed as

$$
\rho\left(\mathbf{x}, y=\frac{\left.\sum_{i} \sum_{j} I(i, j) I(i+x, j+y)\right)}{\sum_{i} \sum_{j} I^{2}(i, j)}\right.
$$

The features computed from autocorrelation matrix are (i) Coarseness $\left(C_{s}\right)$ and (ii) Busyness $\left(B_{s}\right)$ which are computed as follows:

- Coarseness $\left(C_{s}\right)$

$$
C_{s}=\frac{2}{\frac{\sum_{i} \sum_{j} \operatorname{Max}(i, j)}{n}+\frac{\sum_{i} \sum_{j} \operatorname{Max}(i, j)}{m}}
$$

where $\operatorname{Max}(i, j)=1$ if point $(i, j)$ is either a row maxima or column maxima, else $\operatorname{Max}(i, j)=0$.

- Busyness $\left(B_{s}\right)$

$$
B_{g}=1-C_{s}^{\frac{1}{\alpha}}
$$

where $\frac{1}{\alpha}$ is a power to make $C_{s}$ significant against 1 .

\section{Classification}

According to the classical rule of thumb, the number of training patterns for each class must be 5 to 10 times the dimensionality of the feature vector. But due to unavailability of sufficient data, we have had to make do with a far smaller training set. The training data consists of 50 samples and test data consists of 34 samples, with fair representation from each class, but for the exception of the class basophil, of whose we have very few samples. The test instances are different from the ones used for training. We studied the suitability of the features for our task, with respect to various supervised classifiers. Table 1 shows the performance of the classifiers used on various combinations of feature sets.

\section{RESULTS}

The novelty of the approach for automation of $\mathrm{DBC}$ lies in the segmentation scheme used. The proposed segmentation scheme has been applied on 115 peripheral blood smear slides, stained using May-Grunwald-Giesma (MGG) stain. Each image consists of one or more cells of the same or different types, and is of size $1000 \times 1300$. A segmentation accuracy of $80 \%$ is obtained, with hand-marked segmentation

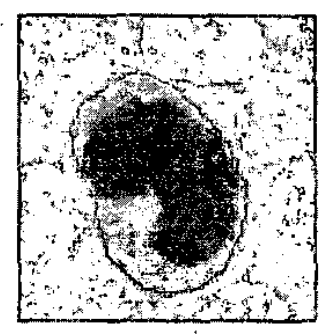

(ai)

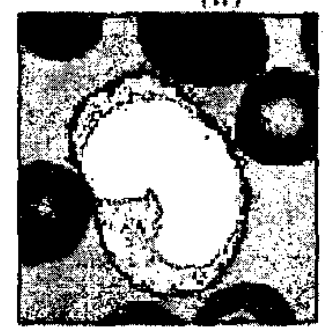

(c)

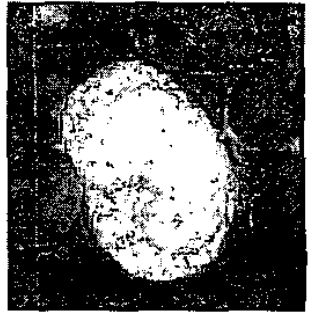

(li)

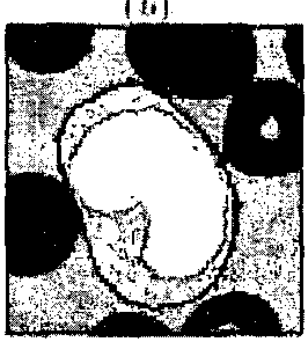

(1)
Figure 2. Sequence of processing (a) Original image (b) Saturation image (c) K-Means output (d) Final output

held as the reference. This scheme is automatic since it needs no parameter-tuning. It would work on any dataset whose magnification is known. For our calculations, all input values are between 0 and 1 . It takes about 20 iterations for the EM: algorithm to converge to an error threshold of 0.00001 . In cases where two non-touching cells appear in the same rectangular patch, care is taken to retain only one at a time. However, if the cells happen to be touching, our system doesn't distinguish them as two different cells. This would need the cells to be recognized as clustered, and a declustering technique needs to be subsequently used. For testing the performance of the system nearly equal samples are chosen from all classes so as to give equal weightage to each class. The best classification accuracy of $97 \%$ is obtained using Neural networks, followed by $94 \%$ using SVM. The classification accuracy obtained using very simple features, is comparable with that obtained with the best of the existing techniques that use more sophisticated features.

Table 1. Comparison of Classifier performance on the feature sets (in \%)

\begin{tabular}{|c||c|c|c|}
\hline Classifier & Texture & Shape-Color & Combined \\
\hline \hline NN & 50.0 & 64.7 & 73.5 \\
\hline KNN & 41.2 & 67.6 & 70.6 \\
\hline W-KNN & 50.0 & 70.6 & 82.3 \\
\hline Bayes & 44.1 & 79.4 & 82.3 \\
\hline SVM & 50.0 & 91.1 & $\mathbf{9 4 . 1}$ \\
\hline NNet $>$ & $\mathbf{7 3 . 5}$ & $\mathbf{9 7 . 1}$ & $\mathbf{9 4 . 1}$ \\
\hline
\end{tabular}




\section{Medical Image Processing / 551}

\section{CONCLUSIONS}

We have developed an efficient automatic system for differential blood count using color images of blood smears. This system requires no user-interaction or parameter tuning, which clearly places it above most techniques conventionally used. The a priori knowledge of the number of classes is very crucial, which remains constant over a given dataset because the nature of the smear images can't vary drastically. The segmentation scheme used in the system can be easily adapted for any given data set with a known magnification. However, in order to be clinically useful, the technique needs to be enhanced to handle clustered cells. The features extracted are very simple and do not require intensive computations. Classification carried out on standard classifiers have yielded results comparable to or better than those claimed with the existing methods.

\section{ACKNOWLEDGEMENTS}

The authors thank Prof. Pandit and Prof. Link, University of Kaiserslautern, Germany, for providing the data.

\section{REFERENCES}

[1] Luc Vincent and Pierre Soille, "Watersheds in digital space and efficient algorithm based on immersion simulations," IEEE Trans. on PAMI,13(6), 583-598, 1991.

[2] Dacie and Lewis, Practical Haematology, Churchill Livingstone Inc., 2001.

[3] R. C. Gonzalez and R. E. Woods, Digital Image Processing, Addison Wesley Publishing Co, 1993.

[4] M. Tuceryan and A. K. Jain, "Texture Analysis," Handbook of Pattern Recognition and computer Vision (2nd Edition), World Scientific Publishing Co.",1998.

[5] J: Park and J. Keller, "Fuzzy Patch Label Relaxation in Bone Marrow Cell Segmentation," A Computational Intelligence System for Cell Classification,pp. 1133-1138, 1997.

[6] ' Yair Weiss, "Motion Segmentation using EM- a short tutorial," Technical Report, MIT, MA 02139, USA,1996.

[7] S. F. Bikhet and A. M. Darwish and H. A. Tolba and S. I. Shaheen, "Segentation and Classification of White Blood Cells," IEEE Intl. Conf. On Acoustics, Speech and Signal Processing, ICASSP,pp. 2259-2261, 1999.

[8] I. Cseke, "A fast segmentation scheme for white blood cell images," 11th IAPR Int. Conf. on Pattern Recognition,Image, Speech and Signal Analysis, pp: 530-533, 1992.

[9] Vassili A.Kovalev and Andrei Y.Grigoriev and Hyo-Sok Ahn, "Robust Recognition of White Blood Cell Images ,"Int. Confon Pattern Recognition, pp. 371-375, 1996.

[10] D.Wermser and G.Haussman and C.E. Liedtke, "Segmentation of blood smears by hierarchical thresholding," Computer Vision, Graphics and Image Processing, pp. $151-168,1984$.

[11] Guclu Ongun and Ugur Halici and Kemal Leblebicioglu and Volkan Atalay, "Feature Extraction and Classification of Blood Cells for an Automated Differential Blood Count System," IEEE IJCNN,pp. 2461-2466, 2001

[12] N. Abbadeni, "Computational Measures Corresponding to Perceptual Textural Features," Proceeding of the IEEE International Conference on Image Processing , pp. $897-900,2000$. 